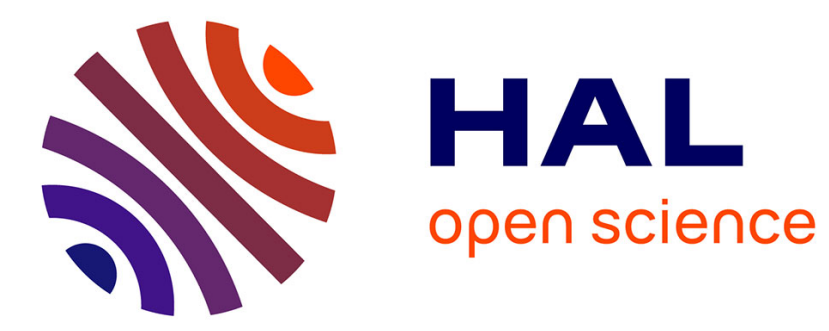

\title{
Inverse parametric convex programming problems via convex liftings
}

Ngoc Anh Nguyen, Sorin Olaru, Pedro Rodriguez-Ayerbe, Morten Hovd, Ion

Necoara

\section{- To cite this version:}

Ngoc Anh Nguyen, Sorin Olaru, Pedro Rodriguez-Ayerbe, Morten Hovd, Ion Necoara. Inverse parametric convex programming problems via convex liftings. 19th World Congress of the International Federation of Automatic Control - IFAC 2014, Aug 2014, Cape Town, South Africa. 10.3182/20140824-6-ZA-1003.02364 . hal-01086492

\section{HAL Id: hal-01086492 \\ https://hal-centralesupelec.archives-ouvertes.fr/hal-01086492}

Submitted on 22 May 2015

HAL is a multi-disciplinary open access archive for the deposit and dissemination of scientific research documents, whether they are published or not. The documents may come from teaching and research institutions in France or abroad, or from public or private research centers.
L'archive ouverte pluridisciplinaire HAL, est destinée au dépôt et à la diffusion de documents scientifiques de niveau recherche, publiés ou non, émanant des établissements d'enseignement et de recherche français ou étrangers, des laboratoires publics ou privés. 


\title{
Inverse parametric convex programming problems via convex liftings
}

\author{
N. A. Nguyen, S. Olaru, P. Rodriguez-Ayerbe ${ }^{*}$ M. Hovd ${ }^{* *}$ I. Necoara ${ }^{* * *}$ \\ * E3S (Supélec Systems Sciences, Automatic Control Department, \\ Gif-sur-Yvette, France (e-mail: Ngocanh.Nguyen, Sorin.Olaru, \\ Pedro.Rodriguez-Ayerbe @ supelec.fr). \\ ** Department of Engineering Cybernetics, Norwegian University of Science \\ and Technologies, Trondheim, Norway (e-mail: morten.hovd@itk.ntnu.no) \\ *** Politehnica University of Bucharest, Automatic Control and Systems \\ Engineering Department, Bucharest, Romania (e-mail: \\ ion.necoara@acse.pub.ro)
}

\begin{abstract}
The present paper introduces a procedure to recover an inverse parametric linear or quadratic programming problem from a given liftable polyhedral partition over which a continuous piecewise affine function is defined. The solution to the resulting parametric linear problem is exactly the initial piecewise affine function over the given original parameter space partition. We provide sufficient conditions for the existence of solutions for such inverse problems. Furthermore, the constructive procedure proposed here requires at most one supplementary variable in the vector of optimization arguments. The principle of this method builds upon an inverse map to the orthogonal projection, known as a convex lifting. Finally, we show that the theoretical results has a practical interest in Model Predictive Control (MPC) design. It is shown that any linear Model Predictive Controller can be obtained through a reformulated MPC problem with control horizon equal to two prediction steps.
\end{abstract}

\section{INTRODUCTION}

Inverse parametric convex programming (PCP) problems are getting increased attention in the scientific community Hempel et al. [2012], Hempel et al. [2013], Baes et al. [2008], Stefano and Bemporad [2010]. One domain of application of these approaches is the complexity reduction of control laws based on parametric linear or quadratic programming problems. To clarify the relevance of considering the inverse parametric linear or quadratic programming problems in constrained model predictive control for example, let us review a generic definition of such control design.

A model predictive control problem aims to minimize a cost function over a finite prediction horizon $N \in \mathbb{N}_{+}$:

$$
F\left(U, x_{k}\right)=\sum_{i=0}^{N-1} \ell_{i}\left(x_{k+i \mid k}, u_{k+i \mid k}\right)+V_{N}\left(x_{k+N \mid k}\right),
$$

where $x_{k} \in \mathbb{R}^{d_{x}}$ is state variable, $u_{k} \in \mathbb{R}^{d_{u}}$ is the control variable and

$$
U=\left[\begin{array}{lll}
u_{k \mid k}^{T} & \cdots & u_{k+N-1 \mid k}^{T}
\end{array}\right]^{T} .
$$

$\ell_{i}\left(x_{k+i \mid k}, u_{k+i \mid k}\right)$ represents a stage cost for $\forall i \in \mathcal{I}_{N-1} \cup\{0\}$ and $V_{N}\left(x_{k+N \mid k}\right)$ denotes a terminal cost function. ${ }^{1}$

This optimization problem is solved in the presence of constraints:

$$
\begin{aligned}
H_{x}^{(i)} x_{k+i \mid k}+H_{u}^{(i)} u_{k+i \mid k} & \leq k^{(i)} \text { for } 0 \leq i \leq N-1 \\
H_{x}^{(N)} x_{k+N \mid k} & \leq k^{(N)}
\end{aligned}
$$

\footnotetext{
1 the following definition is necessary for our introduction and the development of this article for a given $N \in \mathbb{N}_{+}: \mathcal{I}_{N}:=\left\{i \in \mathbb{N}_{+} \mid i \leq N\right\}$.
}

where the matrices $H_{x}^{(i)}, H_{u}^{(i)}$ describe mixed state and input constraints for each stage of prediction horizon. In addition in the linear MPC literature, $\ell_{i}\left(x_{k+i \mid k}, u_{k+i \mid k}\right)$ for $\forall i \in \mathcal{I}_{N-1} \cup$ $\{0\}$ and $V_{N}\left(x_{k+N \mid k}\right)$ have one of the following forms:

(1) quadratic stage and terminal cost:

$$
\begin{aligned}
\ell_{i}\left(x_{k+i \mid k}, u_{k+i \mid k}\right) & =\left\|Q_{i} x_{k+i \mid k}\right\|_{2}^{2}+\left\|R_{i} u_{k+i \mid k}\right\|_{2}^{2}, \\
V_{N}\left(x_{k+N \mid k}\right) & =\left\|P x_{k+N \mid k}\right\|_{2}^{2},
\end{aligned}
$$

(2) a $1 / \infty$-norm stage and terminal cost:

$$
\begin{aligned}
\ell_{i}\left(x_{k+i \mid k}, u_{k+i \mid k}\right) & =\left\|Q_{i} x_{k+i \mid k}\right\|_{p}+\left\|R_{i} u_{k+i \mid k}\right\|_{p}, \\
V_{N}\left(x_{k+N \mid k}\right) & =\left\|P x_{k+N \mid k}\right\|_{p},
\end{aligned}
$$

where $p=1 / \infty$ and $P, Q_{i}, R_{i}$ are full column rank matrices.

The solution to such a problem may be obtained via parametric convex programming:

$$
\begin{aligned}
U^{*} & =\underset{U}{\operatorname{argmin}} F\left(U, x_{k}\right), \\
\text { s.t: } \quad G U & \leq W+E x_{k} .
\end{aligned}
$$

In the implementation, the interest of the optimal solution for the problem above is restricted to the first part of the optimal control sequence: $u_{k}=U^{*}\left(1: d_{u}, \cdot\right)$, and has been shown in Bemporad et al. [2002] to have a piecewise affine feedback structure.

Through the developments of the present paper, we will show that in fact every continuous piecewise affine controller can be recovered via a model predictive control problem at most in horizon $2(N=2)$. Before entering the main developments, let us review some interesting existing results related to the inverse parametric convex programming problems. 
First of all, let us stress that the above claim is not revolutionary from the inverse optimality point of view, noting that in Baes et al. [2008], the authors proved that "every continuous feedback law can be obtained by PCP". The result in Baes et al. [2008] is a beautiful structural result but remains mainly a theoretical one. Indeed, it does not provide neither a constructive procedure nor a qualitative interpretation in terms of dimension for the optimization arguments. Our contribution will be concentrated on these two aspects of the solution for piecewise linear control laws and linear dynamics ${ }^{2}$.

In the present paper, we will prove that one supplementary variable in $\mathbb{R}$ is sufficient for composing a vector of optimization arguments for the solution of inverse problem. Also, the solution of an appropriate linear programming problem leads directly to the given piecewise affine function. The method proposed builds on the lifting which embeds the given polyhedral partition $\mathcal{X} \subset \mathbb{R}^{d_{x}}, d_{x} \in \mathbb{N}_{+}$into a higher dimensional space $\mathbb{R}^{d_{x}+1}$ such that the image of $\mathcal{X}$ via this lifting is a polytope whose orthogonal projection back onto $\mathbb{R}^{d_{x}}$ gives back the given polyhedral partition $\mathcal{X}$. The idea of lifting was studied in the early works of Maxwell, and completed by Crapo, Whiteley, Ceremona in the case $d_{x}=2$, Maxwell [1864], Crapo and Whiteley [1993, 1994]). Such a lifting is called a convex lifting, as the above polytope in $\mathbb{R}^{d_{x}+1}$ is named in this case as affinely equivalent polyhedron to the polyhedral partition $\mathcal{X}$ (more details in Aurenhammer [1987a,b], Schulz [2008], Rybnikov [1999], Aurenhammer [1991], Nguyen et al. [2014]). A procedure to define this parametric linear programming problem with respect to the given polyhedral partition and the known piecewise affine function defined over the above partition will be presented.

\section{NOTATION AND DEFINITIONS}

$\mathbb{R}, \mathbb{R}_{+}, \mathbb{N}_{+}$denote the real number set, the non-negative real number set and the positive integer field, respectively. A polyhedron is defined as the intersection of a finite number of halfspaces in $\mathbb{R}^{n}$. A bounded polyhedral set represents a polytope (more details about the polytope in Grünbaum [1967]). $\mathcal{V}(\cdot)$ defines the vertex set of a bounded polyhedron. If $x$ is an element of the vector space $\mathbb{R}^{d_{x}}, x_{i}$ represents the $i^{t h}$ element of $x$ and belongs to $\mathbb{R}$. We denote $\mathbb{R}_{x}$ the vector space containing $x$ i.e $\mathbb{R}_{x}=\mathbb{R}^{d_{x}}$. For a finite subset $\mathcal{S}=\left\{x_{1}, x_{2}, \ldots, x_{n}\right\} \subset \mathbb{R}^{d_{x}}$, $\operatorname{Card}(\mathcal{S})$ denotes the cardinal number of set $\mathcal{S}$, in other words, $\operatorname{Card}(\mathcal{S})=n$. Moreover, the convex hull of a subset $\mathcal{S} \subset \mathbb{R}^{d_{x}}$ is denoted as $\operatorname{conv}(\mathcal{S})$.

$\operatorname{Proj}_{\mathbb{S}} \mathcal{S}$ presents the orthogonal projection onto the vector space $\mathbb{S}$ of the set $\mathcal{S}$. If $\mathcal{S}$ is a compact bounded subset of $\mathbb{R}^{d_{x}}$, $\operatorname{int}(\mathcal{S})$ represents the relative interior of $\mathcal{S}$. Finally, for a given polyhedral set $\mathcal{S}$, a facet of $\mathcal{S}$ is defined as the intersection of $\mathcal{S}$ and one hyperplane which supports $\mathcal{S}$. In addition, $\mathcal{F}(\mathcal{S})$ presents the set of all facets of $\mathcal{S}$. In the case of a polytope $\mathcal{S} \subset \mathbb{R}^{d_{x}}, \mathcal{F}(\mathcal{S})$ is a finite collection of polytopes of dimension $d_{x}-1$.

Let us consider next some useful definitions.

Definition 2.1. A polyhedral partition of a polytope $\mathcal{X} \subset \mathbb{R}^{d_{x}}$ is defined as follows:

\footnotetext{
2 In Baes et al. [2008] the conclusion section contains the following remark: A natural question that can arise from this note would be to particularize our results to piecewise linear controllers: can any continuous piecewise linear feedback law be obtained by parametric linear programming? Should such a construction be possible, it might offer computational advantages for explicit MPC algorithms. Practically, this problem is addressed in the present paper.
}

(1) $\mathcal{X}=\bigcup_{i \in \mathcal{I}_{N_{r}}} \mathcal{X}_{i}, N_{r} \in \mathbb{N}_{+}$

(2) $\mathcal{X}_{i}$ is polyhedral for $\forall i \in \mathcal{I}_{N_{r}}$

(3) $\operatorname{int}\left(\mathcal{X}_{i}\right) \bigcap \operatorname{int}\left(\mathcal{X}_{j}\right)=\emptyset$ with $i \neq j,(i, j) \in \mathcal{I}_{N_{r}}^{2}$

(4) $\left(\mathcal{X}_{i}, \mathcal{X}_{j}\right)$ are neighbors if $(i, j) \in \mathcal{I}_{N_{r}}^{2}$, $i \neq j$ and $\operatorname{dim}\left(\mathcal{X}_{i} \cap \mathcal{X}_{j}\right)=d_{x}-1$.

Definition 2.2. A function $f_{\text {pwa }}: \mathcal{X} \rightarrow \mathbb{R}^{d_{u}}$ defined over a polyhedral partition $\mathcal{X}=\bigcup_{i \in \mathcal{I}_{N_{r}}} \mathcal{X}_{i} \subset \mathbb{R}^{d_{x}}$ by

$$
\begin{gathered}
f_{p w a}(x)=A_{i} x+a_{i} \text { for } x \in \mathcal{X}_{i} \\
\text { with } A_{i} \in \mathbb{R}^{d_{u} \times d_{x}} \text { and } a_{i} \in \mathbb{R}^{d_{u}}
\end{gathered}
$$

is a piecewise affine function over the partition $\mathcal{X}$.

Definition 2.3. A piecewise affine function $f_{p w a}$ defined in (2.2) is continuous if and only if for $\forall(i, j) \in \mathcal{I}_{N_{r}}^{2}, i \neq j$ such that $\mathcal{X}_{i}, \mathcal{X}_{j}$ are neighbors, then

$$
A_{i} x+a_{i}=A_{j} x+a_{j} \text { for } \forall x \in \mathcal{X}_{i} \cap \mathcal{X}_{j} .
$$

Definition 2.4. A given polyhedral partition $\mathcal{X}=\bigcup_{i \in \mathcal{I}_{N_{r}}} \mathcal{X}_{i} \subset$ $\mathbb{R}^{d_{x}}$ has an affinely equivalent polyhedron if there exists a polytope $\tilde{\mathcal{X}} \subset \mathbb{R}^{d_{x}+1}$ such that for each $i \in \mathcal{I}_{N_{r}}$ :

1) $\exists F_{i} \in \mathcal{F}(\widetilde{\mathcal{X}})$ satisfying: $\operatorname{Proj}_{\mathbb{R}^{d_{x}}} F_{i}=\mathcal{X}_{i}$,

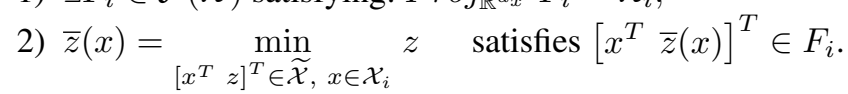

Remark 2.5. The second condition in the definition of an affinely equivalent polyhedron ensures that the set of facets of $\widetilde{\mathcal{X}}$ at the lower values of $z$ are exclusively considered. The image of these facets via the orthogonal projection is the polyhedral partition $\mathcal{X}$.

Remark 2.6. In this article, a polyhedral partition having an affinely equivalent polyhedron is called a liftable polyhedral partition.

In order to introduce a projection able to recover a polyhedral partition from an affinely equivalent polyhedron, we introduce a new operator called partitioned orthogonal projection.

Definition 2.7. Given a polytope $\widetilde{\mathcal{X}} \subset \mathbb{R}^{d_{x}+1}$, the partitioned orthogonal projection of $\widetilde{\mathcal{X}}$ on the first $d_{x}$ coordinates is denoted as $X \operatorname{proj}_{\mathbb{R}^{d_{x}}} \widetilde{\mathcal{X}}$, and is mathematically defined as:

$$
\begin{aligned}
& \operatorname{Xproj}_{\mathbb{R}^{d_{x}}} \tilde{\mathcal{X}}:=\left\{\bigcup_{i \in \mathcal{I}_{N_{r}}} P_{i} \subset \mathbb{R}^{d_{x}} \mid\right. \\
& \text { with } P_{i}=\operatorname{Proj}_{\mathbb{R}^{d_{x}}} F_{i}, \quad F_{i} \in \mathcal{F}(\widetilde{\mathcal{X}}), \\
& \left.\left[\begin{array}{c}
x \\
\bar{z}(x)
\end{array}\right] \in F_{i}, \quad \forall x \in P_{i} \text { and } \bar{z}(x)=\min _{\left[\begin{array}{ll}
x^{T} & z
\end{array}\right]^{T} \in \widetilde{\mathcal{X}}, x \in P_{i}} z\right\} \text {. }
\end{aligned}
$$

Remark 2.8. $X \operatorname{proj}_{\mathbb{R}^{d_{x}}} \widetilde{\mathcal{X}}$ is a finite collection of polyhedral sets by the fact that $\mathcal{F}(\widetilde{\mathcal{X}})$ is a finite union of sets in $\mathbb{R}^{d_{x}+1}$. The set of facets $F_{i}, i \in \mathcal{I}_{N_{r}}$ of $\widetilde{\mathcal{X}}$ are continuous, and they compose a convex surface in $\mathbb{R}^{d_{x}+1}$. In addition the continuity property of this surface is inherited via the orthogonal projection by $X \operatorname{proj}_{\mathbb{R}^{d_{x}}} \widetilde{\mathcal{X}}$ which is a polyhedral partition. The uniqueness of the partitioned orthogonal projection is related to the uniqueness of the set of facets $\mathcal{F}(\widetilde{\mathcal{X}})$. 


\section{PARAMETRIC LINEAR/QUADRATIC PROGRAMMING PROBLEM}

The control law design based on parametric linear/quadratic programming has been studied extensively in the last decade, its solution is a continuous piecewise affine function defined over a state space partition Bemporad et al. [2002], Seron et al. [2003], Tøndel et al. [2003], Olaru and Dumur [2005]. With $d_{x}, d_{U} \in \mathbb{N}_{+}$, its compact formulation is the following:

$$
\min _{U} f(U, x)
$$

such that $G U \leq W+E x, \quad x \in \mathbb{R}^{d_{x}} \quad U \in \mathbb{R}^{d_{U}}$,

where $f(U, x)$ presents a linear or quadratic cost function of $U$, $U \in \mathbb{R}^{d_{U}}$ being the decision variable, $x \in \mathbb{R}^{d_{x}}$ being the vector of parameters, the above problem has a continuous solution in which the parameter space partition is a polyhedral partition,

$$
\mathcal{X}=\bigcup_{i \in \mathcal{I}_{N_{r}}} \mathcal{X}_{i}
$$

and the decision variable is a continuous piecewise affine function over the above parameter space partition

$$
U=f_{p w a}(x)=F_{i} x+G_{i} \text { for } \forall x \in \mathcal{X}_{i} .
$$

Geometrically, a polyhedral partition can be obtained by the orthogonal projection onto the parameter space of a polytope in higher dimension for the case of linear programming. Notice that for the case of multiparametric quadratic programming, the parameter space partition $\mathcal{X}$ is also the union of the polytopes which are the images via the orthogonal projection of some facets of the polytope in higher dimension characterized by the constraints on decision variables and parameters in the so-called parameterized polyhedra approach Olaru and Dumur [2004]. Naturally, the parameter space in this case remains polyhedral due to the considered linear structure of the constraints. In addition, this parameter partition can be refined in order to obtain a convex liftable according to a recent geometric result in Nguyen et al. [2014]. More precisely, there exist a partition associated with the explicit solution denoted as $\mathcal{X} \subset \mathbb{R}^{d_{x}}$ which is the image via the partitioned orthogonal projection onto the parameter space $\mathbb{R}^{d_{x}}$ of an affinely equivalent polyhedra in $\mathbb{R}^{d_{x}+1}$.

\section{INVERSE PARAMETRIC LINEAR/QUADRATIC PROGRAMMING PROBLEM}

The classical MPC design and the relationships between linear MPC and linear/quadratic multiparametric programming are well understood. There is however a fundamental problem that needs to be answered: provided an MPC controller is functioning adequately, which is the minimal horizon MPC problem, leading to the same feedback control law? The interest in such a minimal horizon MPC formulation is related to the real time complexity issues on one side and the fact that the tail of predicted sequence (2) is implicitly reduced.

Practically this is an inverse optimality problem and its resolution exploits the piecewise affine structure of the MPC controller. From a mathematical point of view, the inverse parametric optimization problem aims to recover an appropriate parametric linear/quadratic programming problem such that for a given polyhedral parameter space partition $\mathcal{X}=$ $\bigcup_{i \in \mathcal{I}_{N_{r}}} \mathcal{X}_{i} \subset \mathbb{R}^{d_{x}}$ and a continuous piecewise affine function $u(x)=f_{p w a}(x)=f_{i} x+g_{i}$ for $\forall x \in \mathcal{X}_{i}$, the optimality condition is fulfilled. Let us briefly state it in the following:
Problem statement: For a given polyhedral partition $\mathcal{X}=$ $\bigcup_{i \in \mathcal{I}_{N_{r}}} \mathcal{X}_{i} \subset \mathbb{R}^{d_{x}}$ and a continuous piecewise affine function $f_{\text {pwa }}: \mathcal{X} \rightarrow \mathbb{R}^{d_{u}}$, find $J(x, u, z), H_{x}, H_{u}, H_{z}, K$ such that

$$
\left\{\begin{aligned}
f_{p w a}(x)=\operatorname{Proj}_{\mathbb{R}^{d_{u}}} \arg \min _{\left[u^{T} z\right]^{T}} J(x, u, z), \\
\text { s.t. } \quad H_{u} u+H_{x} x+H_{z} z \leq K .
\end{aligned}\right.
$$

Let us state some assumptions which seem reasonable for development of the results without a complicated notation and pre-treatment of the input data:

\section{Assumption 4.1 .}

(1) The parametric linear and quadratic programming problems are exclusively considered as possible candidates for the inverse optimality problem. By consequence, the cost function has the following form with $Q^{T}=Q \geq 0$ :

$$
J(x, u, z)=\left[\begin{array}{lll}
x^{T} & u^{T} & z^{T}
\end{array}\right] Q\left[\begin{array}{l}
x \\
u \\
z
\end{array}\right]+C^{T}\left[\begin{array}{l}
x \\
u \\
z
\end{array}\right] .
$$

(2) The given polyhedral partition $\mathcal{X}$ is liftable.

Assumption 1 provides a manageable framework for the constructive inverse optimality procedures. Larger classes of objective functions can provide some additional degrees of freedom but move away from the principles of linear MPC. Assumption 2 underlines the liftablility of the partitions. This condition is sufficient for the existence of a solution and contains a series of geometrical considerations which are beyond the scope of the present paper Nguyen et al. [2014].

The MPC design can be stated using separated polyhedral state and input constraints: $u_{k} \in \mathbb{U}$ and $x_{k} \in \mathbb{X}$. Alternatively, stating mixed input and state constraints can offer a generic framework for linear constraint specification. Additionally, the mixed input-state constraints relax the structural constraints for the construction of an inverse optimality solution. The above remark leads us to the introduction of the invertibility definition.

Definition 4.2. A continuous piecewise affine function defined over a polyhedral partition is called invertible if there exists an appropriate constraint set and a cost function such that the associated parametric convex programming problem has as optimal solution the given continuous piecewise affine function over a given polyhedral partition.

Although the inverse optimality problem is related to the existence of a model predictive control, we will concentrate in the first stage on the mathematical issue, leaving aside the notation and the relationship with the state space and the control variable.

\section{SOLUTION TO THE INVERSE PARAMETRIC LINEAR/QUADRATIC PROGRAMMING PROBLEM}

Our objective is to recover the given parameter space partition as a solution of a multiparametric program. Thus our interest is exclusively focused on the case where there exists an affinely equivalent polyhedron to the given polyhedral partition ${ }^{3}$.

Let us introduce the set of vertices of the regions in the polyhedral partition as:

\footnotetext{
3 Readers can find more details about the non-liftable polyhedral partition in Nguyen et al. [2014]
} 


$$
V_{x}=\bigcup_{i \in \mathcal{I}_{N_{r}}} \mathcal{V}\left(\mathcal{X}_{i}\right),
$$

and denote the cardinal of this finite set as $n=\operatorname{Card}\left(V_{x}\right)$. Thus $V_{x}$ can be rewritten as:

$$
V_{x}=\left\{x^{(1)}, \ldots, x^{(n)}\right\} .
$$

Starting from the liftability hypothesis, let $\Pi_{\left[x^{T} z\right]^{T}} \subset \mathbb{R}^{d_{x}+1}$ be an affinely equivalent polyhedron to the given parameter space polyhedral partition $\mathcal{X}$ with the extended variable $z \in \mathbb{R}$ obtained for example via the constructive procedure in Nguyen

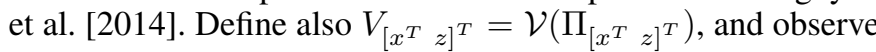
that $\left.V_{\left[x^{T}\right.} z\right]^{T}$ has the form:

$$
\left.V_{\left[x^{T}\right.} z\right]^{T}=\left\{\left[\begin{array}{l}
x^{(1)} \\
z^{(1)}
\end{array}\right], \ldots,\left[\begin{array}{l}
x^{(n)} \\
z^{(n)}
\end{array}\right]\right\} .
$$

Let us state an intermediate result for our constructive inverse optimality problem resolution:

Proposition 5.1. Given $\Gamma_{s} \subset \mathbb{R}^{d_{s}}$ a convex polyhedron such that $\mathcal{V}\left(\Gamma_{s}\right)=\left\{s^{(1)}, \ldots, s^{(q)}\right\}$. For any finite set of points $\left\{t^{(1)}, \ldots, t^{(q)}\right\} \subset \mathbb{R}^{d_{t}}$ not lying on a hyperplane, an extension of the family $\mathcal{V}\left(\Gamma_{s}\right)$ can be obtained in higher dimensional space $\mathbb{R}^{d_{s}+d_{t}}$ for the vectors $\left[s^{T} t^{T}\right]^{T}$ in order to obtain the set:

$$
V_{\left[s^{T} t^{T}\right]^{T}}:=\left\{\left[\begin{array}{l}
s^{(1)} \\
t^{(1)}
\end{array}\right], \ldots,\left[\begin{array}{l}
s^{(q)} \\
t^{(q)}
\end{array}\right]\right\},
$$

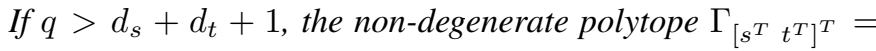
$\operatorname{conv}\left(V_{\left[s^{T} t^{T}\right]^{T}}\right)$ satisfies:

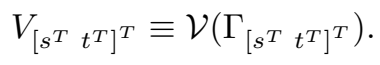

Based on the above proposition, the solution to an inverse parametric linear or quadratic programming problem can be stated as follow:

Theorem 5.2. Let a continuous piecewise affine function $u(x)=$ $f_{\text {pwa }}(x) \in \mathbb{R}^{d_{u}}$ be defined over a liftable polyhedral partition $\mathcal{X}=\bigcup_{i \in \mathcal{I}_{N_{r}}} \mathcal{X}_{i} \subset \mathbb{R}^{d_{x}}$. Then, there exists $\Pi_{\left[x^{T} z\right]^{T}}$ an affinely equivalent polyhedron in $\mathbb{R}^{d_{x}+1}$ to $\mathcal{X}$ where $z \in \mathbb{R}$ denotes the

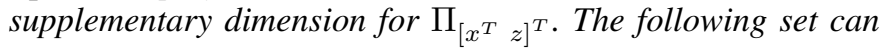
be defined:

$$
\begin{aligned}
& \left.\left.V_{\left[x^{T}\right.} z\right]^{T}=\mathcal{V}\left(\Pi_{\left[x^{T} z\right.}\right]^{T}\right),
\end{aligned}
$$



$$
\begin{aligned}
& \left.V_{\left[x^{T}\right.} z u^{T}\right]^{T}=\left\{\left[\begin{array}{c}
x^{(1)} \\
z^{(1)} \\
f_{p w a}\left(x^{(1)}\right)
\end{array}\right], \ldots,\left[\begin{array}{c}
x^{(q)} \\
z^{(q)} \\
f_{p w a}\left(x^{(q)}\right)
\end{array}\right]\right\},
\end{aligned}
$$

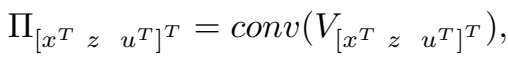

and the following properties hold true:

(1)

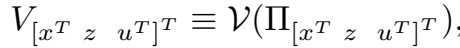

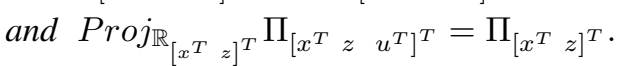

(2) The given piecewise affine function $u=f_{p w a}(x)$ is the image via the orthogonal projection into $\mathbb{R}^{d_{u}}$ of the solution to the below optimization problem:

$$
\begin{array}{cc} 
& \left.\min _{\left[u^{T}\right.} z\right]^{T} \\
\text { such that } & {\left[\begin{array}{lll}
x^{T} & z & u^{T}
\end{array}\right]^{T} \in \Pi_{\left[\begin{array}{lll}
x^{T} & z & u^{T}
\end{array}\right]^{T} .}}
\end{array}
$$

At this moment, we can summarize our constructive procedure towards recovering a given parameter space polyhedral partition over which a continuous piecewise affine function is defined.

\section{Algorithm 2}

Assumption: The existence of a continuous piecewise affine function $u_{p w a}(x)$ defined over a liftable polyhedral partition $\mathcal{X}=\bigcup_{i \in \mathcal{I}_{N_{r}}} \mathcal{X}_{i} \subset \mathbb{R}^{d_{x}}$

Solution:

1: Construct an affinely equivalent polyhedron in $\mathbb{R}^{d_{x}+1}$ for $\mathcal{X}$. 2: Formulate a linear programming problem as the one described in theorem 5.2 where the constraints on $x, u, z$ are

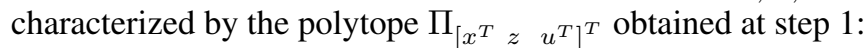

$$
\begin{gathered}
\min _{\left[\begin{array}{ll}
u^{T} & z
\end{array}\right]^{T}} z \\
\text { subject to: }\left[\begin{array}{lll}
x^{T} z & u^{T}
\end{array}\right]^{T} \in \Pi_{\left[\begin{array}{lll}
x^{T} & z & u^{T}
\end{array}\right]^{T}} .
\end{gathered}
$$

3: The given continuous piecewise affine function $u_{p w a}(x)$ is obtained by extracting the first $d_{u}$ coordinates of the optimal solution from the above problem.

Let us now consider some characteristics of our proposed constructive procedure about the invertibility and complexity of such an inverse parametric convex programming problem.

Theorem 5.3. (Invertibility) Given a liftable polyhedral partition $\mathcal{X} \subset \mathbb{R}^{d_{x}}$, any piecewise affine function $u_{\text {pwa }}(x): \mathcal{X} \rightarrow$ $\mathbb{R}^{d_{u}}$ is invertible if $u_{\text {pwa }}(x)$ is continuous.

Theorem 5.4. (complexity) The solution of any multiparametric linear/quadratic programming problem can be obtained by a parametric linear programming problem with at most one supplementary 1-dimensional variable.

\section{RELATED MODEL PREDICTIVE CONTROL PROBLEMS}

In this section, the related model predictive control problems will be presented as applications of the aforementioned constructive procedure of inverse optimal solutions. From the practical point of view in control system theory, the state variable is considered as a parameter, the control action is presented as the decision variable. It is now the time to investigate some results related to invertibility and complexity of a MPC problem.

Theorem 6.1. (inverse optimality of a continuous PWA controller) Any continuous piecewise affine control law defined over a liftable polyhedral partition in the state space can be obtained through a parametric linear programming problem.

Central to the following result is the complexity of such an inverse MPC problem.

Theorem 6.2. (complexity of a MPC problem) The explicit continuous solution of a generic linear/quadratic MPC problem can be obtained through another linear MPC problem having the control horizon at most equal to 2 prediction steps.

Proof: The explicit continuous solution to a MPC problem has the following form:

$$
\begin{aligned}
\mathcal{X} & =\bigcup_{i \in \mathcal{I}_{N_{r}}} \mathcal{X}_{i} \subset \mathbb{R}^{d_{x}} \quad N_{r} \in \mathbb{N}_{+}, \\
u_{k} & =f_{\text {pwa }}\left(x_{k}\right)=f_{i} x_{k}+g_{i} \in \mathbb{R}^{d_{u}} \text { for } \quad \forall x_{k} \in \mathcal{X}_{i} .
\end{aligned}
$$

If $\mathcal{X}$ is not liftable, the regions of the partition $\mathcal{X}$ have to be refined without modifying the PWA function in order to ensure the liftability properties. This is possible via an adequate 
subpartition called an arrangement of hyperplanes ${ }^{4}$. As such we will focus on liftable partition in the following.

Let $\left.\Pi_{\left[x_{k}^{T}\right.} z\right]^{T}$ be an affinely equivalent polyhedron to $\mathcal{X}$. Recall that $z \in \mathbb{R}$ and with $n \in \mathbb{N}_{+}$:

$$
\mathcal{V}\left(\Pi_{\left[\begin{array}{ll}
x_{k}^{T} & z
\end{array}\right]^{T}}\right):=\left\{\left[\begin{array}{c}
x_{k}^{(1)} \\
z^{(1)}
\end{array}\right], \ldots,\left[\begin{array}{c}
x_{k}^{(n)} \\
z^{(n)}
\end{array}\right]\right\} .
$$

With any values of $s^{(1)}, \ldots, s^{(n)} \in \mathbb{R}^{d_{u}-1}$ which do not lie on the same hyperplane, by Proposition 5.1 we obtain:

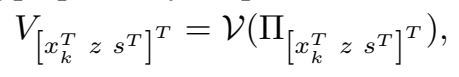

where,

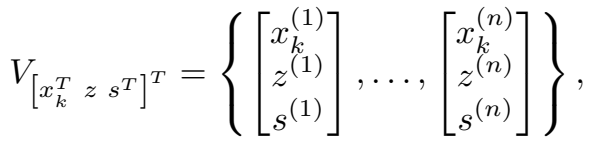

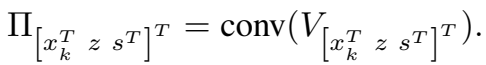

Imposing $u_{k+1 \mid k}^{(i)}=\left[z^{(i)} s^{(i)^{T}}\right]^{T} \in \mathbb{R}^{d_{u}}, u_{k}^{(i)}=f_{p w a}\left(x_{k}^{(i)}\right)$ for $\forall i \in \mathcal{I}_{n}$, one can construct the set of constraints on $x_{k}, u_{k}, u_{k+1 \mid k}$ :

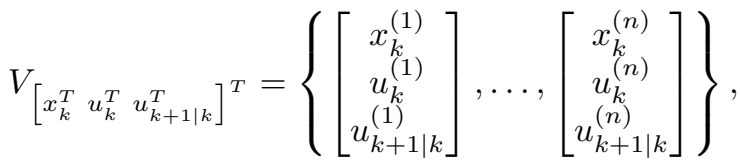

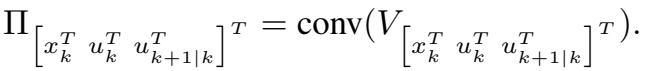

Moreover, a cost function can be chosen as follow:

$$
\begin{aligned}
& l_{0}\left(x_{k}, u_{k}\right)=0, \quad V_{N}\left(x_{k+2 \mid k}\right)=0 . \\
& l_{1}\left(x_{k+1 \mid k}, u_{k+1 \mid k}\right)=u_{k+1 \mid k}(1)=z,
\end{aligned}
$$

Through Theorem 5.2, it is straightforward to see that the MPC problem characterized by constraint set (27) and cost function (28) gives back (24).

\section{NUMERICAL EXAMPLE}

In order to illustrate the proposed constructive procedure, let us consider the double integrator system with sample time $T_{e}=0.5$ :

$$
\begin{aligned}
x_{k+1} & =\left[\begin{array}{cc}
1 & 0.5 \\
0 & 1
\end{array}\right] x_{k}+\left[\begin{array}{c}
0.125 \\
0.5
\end{array}\right] u_{k}, \\
y_{k} & =\left[\begin{array}{ll}
1 & 0
\end{array}\right] x_{k} .
\end{aligned}
$$

A cost function over prediction horizon $N=5$ to be minimized can be presented as follows with respect to weighting matrices $Q=\left[\begin{array}{cc}10 & 0 \\ 0 & 10\end{array}\right], R=0.5$ :

$J=\sum_{i=0}^{4}\left(x_{k+i \mid k}^{T} Q x_{k+i \mid k}^{T}+u_{k+i \mid k}^{T} R u_{k+i \mid k}\right)+x_{k+5 \mid k}^{T} P x_{k+5 \mid k}$,

where $P$ is computed from the Riccati equation. The constraints on control variable and state variable at the present time are: $u_{k} \in\left[\begin{array}{ll}-2 & 2\end{array}\right]$ and $\left[\begin{array}{cc}1 & 0 \\ -1 & 0\end{array}\right] x_{k} \leq\left[\begin{array}{l}5 \\ 5\end{array}\right]$.

The feedback control law is depicted in Figure 2, its associated partition $\mathcal{X}$ is showed in Figure 1 . The facets of an affinely equivalent polyhedron to $\mathcal{X}$ whose projection into $\mathbb{R}_{x}$ is the given state space partition is illustrated in Figure 3. Finally, the

\footnotetext{
4 A discussion on these topics can be found in Nguyen et al. [2014].
}

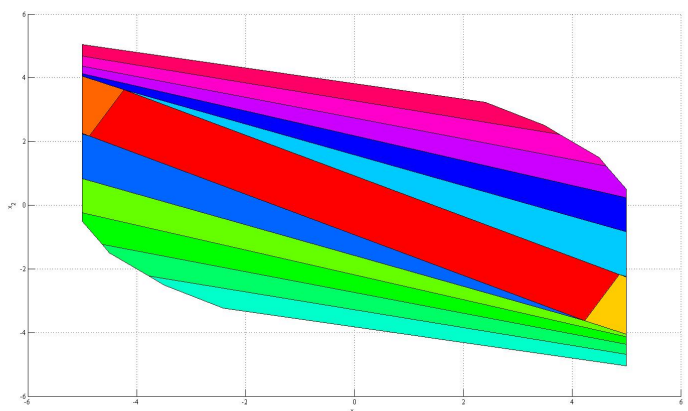

Fig. 1. The polyhedral state space partition $\mathcal{X}$.

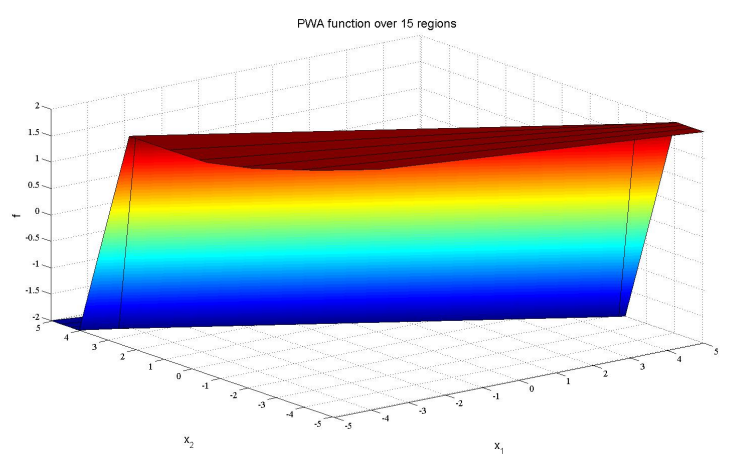

Fig. 2. The piecewise affine controller that we aim to recover.

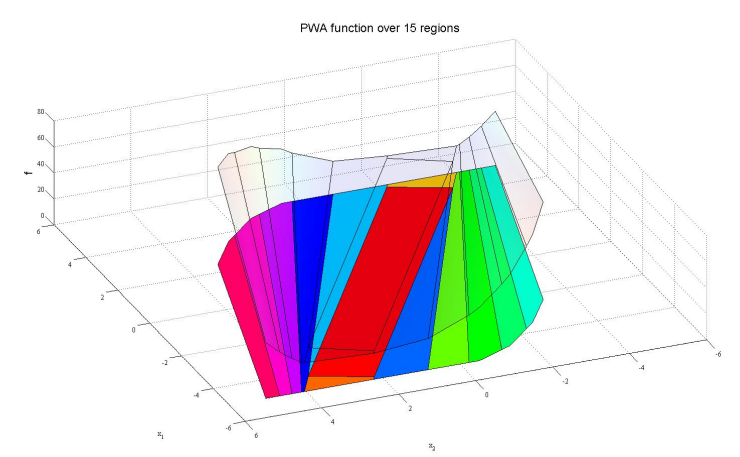

Fig. 3. The collection of facets of an affinely equivalent polyhedron of $\mathcal{X}$ whose orthogonal projection into $\mathbb{R}^{d_{x}}$ leads to $\mathcal{X}$.

result of parametric linear programming problem constructed via Algorithm 2 is shown in Figure 4. As it can be observed that the PWA controllers in Figure 2 and in Figure 4 are equivalent.

\section{CONCLUSIONS AND RELATED WORKS}

The present paper provides a constructive procedure to recover an inverse parametric convex programming problem with respect to a given polyhedral partition over which a continuous piecewise affine function defined. It was shown that the convex lifting can be used as the main methodological concept, thus bringing the inverse optimality problem to a simple geometric structure that can subsequently be linked to a linear programming formulation. Its application is directly linked to model predictive control, where the polyhedral partition stems from the partitioning of the feasible region of the state space into critical regions for different active sets of constraints, and the 


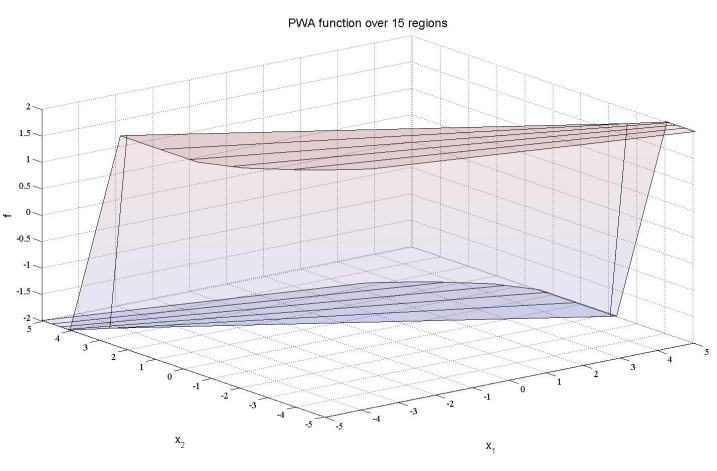

Fig. 4. The result of parametric linear programming problem built on Algorithm 2 for the double integrator system. The equivalent MPC problem has a prediction horizon equal to two prediction steps.

continuous piecewise affine function corresponds to the piecewise affine feedback control law. This offers opportunities to adjust the complexity of MPC design by the reduction of the tail of the predicted control sequences.

Recently, two papers reported a series of developments on a closely related topic (hybrid systems represented via PWA dynamical systems), building essentially on a similar inverse optimality argument. In Hempel et al. [2012], the authors recover a parametric quadratic programming problem with a set of constraints on decision variables and parameters, and a quadratic cost function for the fulfillment of optimality conditions. Subsequently, in Hempel et al. [2013] a method to construct a linear programming problem whose solution is equivalent to a given piecewise affine function has been also presented. It builds on the decomposition of a continuous piecewise affine function into the difference of two continuous convex functions. They showed also that the number of supplementary variables in $\mathbb{R}$ is at most $d_{u}$, where $\operatorname{dim}\left(u_{k}\right)=d_{u}, u_{k}$ is the control variable. All these developments follow a different approach than the one presented in the present paper. We emphasize also that in our theoretical results, there is only one supplementary dimension added to the vector of arguments of the optimization problem. Also, the application of the inverse optimal problem in the case of our results is intended for the MPC design for LTI systems. In this direction, we provide a result on the complexity of such synthesis.

\section{REFERENCES}

F. Aurenhammer. Criterion for the affine equivalence of cell complexes in $r^{d}$ and convex polyhedra in $r^{d+1}$. Discrete and Computational Geometry, 2:49-64, 1987a.

F. Aurenhammer. Recognising polytopical cell complexes and constructing projection polyhedra. Journal of Symbolic Computation, 3:249-255, 1987b.

F. Aurenhammer. Voronoi diagrams: A survey of a fundamental data structure. ACM Comput. Surveys, 23:345-405, 1991.

M. Baes, M. Diehl, and I. Necoara. Every continuous nonlinear control system can be obtained by parametric convex programming. IEEE tran on Automatic Control, 53(8):19631967, Sept 2008.

A. Bemporad, M. Morari, Vivek Dua, and Efstratios N Pistikopoulos. The explicit linear quadratic regulator for constrained systems. Automatica, 38(1):3-20, 2002.
H. Crapo and W. Whiteley. Plane self stresses and projected polyhedra 1: the basic pattern. Structural Topology, 19:5573, 1993.

H. Crapo and W. Whiteley. Spaces of stresses, projections and parallel drawings for spherical polyhedra. Contributions to Algebra and Geometry, 35(So. 2):259-281, 1994.

B. Grünbaum. Convex polytopes. Wiley Interscience, 1967.

A. B. Hempel, P. J. Goulart, and J. Lygeros. Inverse parametric quadratic programming and an application to hybrid control. In 4th IFAC Nonlinear Model Predictive Control Conference International Federation of Automatic Control Noordwijkerhout, NL. August 23-27, 2012.

A. B. Hempel, P. J Goulart, and J. Lygeros. Every continuous piecewise affine function can be obtained by solving a parametric linear program. In European Control Conference July 17-19, Zurich, Switzerland, 2013.

J. C. Maxwell. On reciprocal diagrams and diagrams of forces. Philosophical Magazine, ser. 4. 27:250-261, 1864.

N. A. Nguyen, S. Olaru, P. Rodriguez-Ayerbe, M. Hovd, and I. Necoara. On the lifting problems and their connections with piecewise affine control design. In European Control Conference, Strasbourg, France, 2014.

S. Olaru and D. Dumur. A parameterized polyhedra approach for explicit constrained predictive control. In Decision and Control, 2004. CDC. 43rd IEEE Conference on, volume 2, pages 1580-1585, 2004.

S. Olaru and D. Dumur. Avoiding constraints redundancy in predictive control optimization routines. Automatic Control, IEEE Transactions on, 50(9):1459-1465, 2005.

K. Rybnikov. Polyhedral Partitions and Stresses. PhD thesis, Queen University, Kingston, Ontario, Canada, 1999.

A. Schulz. Lifting Planar Graphs to Realize Integral 3Polytopes and Topics in Pseudo-Triangulations. PhD thesis, Fachbereich Mathematik und Informatik der Freien Universitat Berlin, 2008.

M. M. Seron, G. C. Goodwin, and J. A. Doná. Characterisation of receding horizon control for constrained linear systems. Asian Journal of Control, 5(2):271-286, 2003.

D. C. Stefano and A. Bemporad. Model predictive control tuning by controller matching. Automatic Control, IEEE Transactions on, 55(1):185-190, 2010.

P. Tøndel, T. A. Johansen, and A. Bemporad. An algorithm for multi-parametric quadratic programming and explicit mpc solutions. Automatica, 39(3):489-497, 2003. 